Monatsschrift f. Geburtshülfe u. Gynäkologie 1930;85:73-78

\title{
II. Buchbesprechungen
}

H'alban-Seitz, Biologie und Pathologie des Weibes. Band IV. (Fort-

setzung aus Band 84, Heft 5/6.) Fr. Kermauner, Klinik der operativen Behandlung der

Krebs formen der

Gebärmutter.

Symptomatologie, Diagnostik und Prognose des Kollum- und Korpus-karzinoms werden erschöpfend abgehandelt und sind von schönen Ab-bildungen begleitet. Die Technik der abdominellen Operation folgt im wesentlichen den Wertheimschen Angaben mit Benützung mancher von Franz und Bumm angegebenen Modifikationen. Die vaginale Operation wird nach der Scńauíaschen Methode geschildert. Sie ist einstweilen be-kanntlich durch Stoeckels Methode überholt. Sehr verdienstvoll ist die Zu-sammenstellung der statistischen Früh- und Spätergebnisse.

, Eine Pvichtigstellung: Bei der Erörterung der Geschichte der operativen Behandlung sagt Kermauner, daß 1878 W. A. Freund den Vorschlag gemacht habe, die Laparatomie zu wählen und ein halbes Jahr später von Czerny zuerst die vaginale Operation vorgenommen worden sei. In Wirk-lichkeit hat Freund nicht nur einen Vorschlag gemacht, sondern die Operation auch ausgeführt. Die 1878 von ihm abdominell operierte Patientin hat Freund selbst 20 Jahre später auf der Naturforscherversammlung in Breslau in der gynäkologischen Sektion als geheilt vorgestellt und gleich-zeitig die mikroskopischen Präparate des Falles demonstriert.

H. Eymer-Innsbruck, Die Klinik der Bestrahlung der Gebärmutíerkrebse.

Nachdem Seitz die physikalischen Grundlagen der Strahlenbehand-lung im zweiten Band des Handbuchs ausführlich dargelegt hat, gibt Burner hier eine Darstellung der klinischen Seite dieser Therapie des Krebses, die in den letzten 10 Jahren die chirurgische Behandlung weitgehend verdrängt hat. Auf die Darstellung der historischen Entwicklung 74

Buchbesprechmigen.

dieser Behandlung im In- und Ausland folgt die Darlegung der makro-und mikroskopischen Befunde am bestrahlten Gewebe mit Wiedergabe der eindrucksvollen Abbildungen von Dòderlein. Die Technik der Radium-bestrahlung ist im wesentlichen nacli der Methode der Heidelberger Klinik geschildert. Kombinierte Bestrahl·ung mit Radium und Röntgen, Vor-und Nachbestrahlung bei operativer Therapie, Röntgen- und Radium -schädigungen und ihre Verhütung finden eingehende Berücksichtigung. Die Statistik enthält als neuen Beitrag die Ergebnisse der Heidelberger Klinik über 203 Fälle mit $25 \%$ Dauerheilung des Kollumkarzinoms. Auf Grund dieser Ergebnisse tritt Eymer für die Radium-Röntgenbestrahlung auch der operablen Fälle ein.

Halban-Seitz, Biologie und Pathologie des Weibes. Band V, I. Teil. Berlin und Wien, Urban \& Schwarzenberg. H. Dietrich-Göttingen, Die Neubildungen der Eíleíter. Erschöpfende Zusammenstellung mit genauen Literaturangaben.

Th. Heynemann-Hamburg, Die Entzündungen der Adnexe und des Becken-peritoneums. 
Die Häufigkeit der Gonorrhöe und die Zunahme der artefiziellen Aborte erklären die große Zahl und die klinische Bedeutung dieser Er-krankungen, die in manchen Anstalten bis zu 30\% aller Fälle ausmachen. Heynemanns übersichtliche Darstellung zeigt, daß über pathologische Anatomie, klinische Erscheinungen und Diagnose kaum größere Meinungs-verschiedenheiten bestehen. Um so mehr dagegen über die Behandlung. Heynemann vertritt mit durchschlagenden Gründen den mehr und mehr sich durchsetzenden Standpunkt eines weitgehenden Konservatismus, ver-schließt sich aber nicht der Notwendigkeit und Zweckmäßigkeit geeigneter Operationen in ausgesuchten Fallen, wofür er eingehende Indikationen aufstellt. Mit der Injektionstherapie spezifischer und unspezifischer Mittel hat Heynemann, der sich dabei auf über 600 Vergleichsfälle stützen kann, etwa den gleichen Erfolg gehabt wie mit einfacher Bettruhe. L. Nürnberger-Halle, Erkrankungen des Neben-Eierstocks und des übrigen mesonephritischen Systems.

Es sind im wesentlichen Zysten des Epoophorons (Parovarium ist eine philologisch inkorrekte Wòrtbildung), des ITrnierenkörpers und Ur-nierenganges, die hier in Frage kommen. Seltener sind solide Tumoren, Dermoide, Adenomyome und Karzinome. Eigene Beobachtungen und erschöpfende Literaturübersicht.

Derselbe, Aktinomykose, Echinokokken, Bilharzíosís.

Überaus gründliche Abhandlung über diese seltenen Erkrankungen, die aber. doch immer wieder zu diagnostischen Irrtümern Anlaß geben. Bewundernswerte Verarbeitung der hauptsächlich ausländischen Literatur.

W. Weibcl-Wien, Tuberkulose des weiblichen Genitalapparates.

Sehr sympathisch berührt die entschiedene Stellungnahme des Autors zu den zahlreichen Problemen dieser Erkrankung, auch wo man sie nicht zu teilen vermag. Man spurt überall den kritischen und selbständigen Forscher und Kliniker. Besonderes Interesse wird die Schilderung der

Buchbespreehungen.

75

Tuberkulose des graviden und puerperalen Uterus fínden sowie die Ab-bildungen, die den Übergang der Tuberkelbazillen auf Plazenta und Fötus illustrieren. Weibel schließt sich hier den Ergebnissen Sitzenfreys an. Danach kann am Vorkommen einer kongenitalen Tuberkulose des Fötus nicht mehr gezweifelt werden. Bei der viel umstrittenen Erage der Therapie wägt Weibel Vorteile und 1·íachteile der einzelnen Methoden (konservative Behandlung, Operation, Röntgenbestrahlung) eingehend ab und bekennt sich zu einem vorsichtigen Eklektizismus. Sein Standpunkt, fast jeclen Fall von Genitaltuberkulose einem operativen Eingriff zu unterziehen, entweder bloß zur Sicherstellung der Diagnose oder zur Entleerung von angesammelter Flüssigkeit und von käsig-eitrigen Massen, steht damit nicht ganz im Einklang und wird kaum allgemein geteilt werden.

Halban-Seitz, Biologie und Pathologie des Weibes. BandV, IV. Teil. Berlin und Wien, Urban \& Schwarzenberg. ,1. Novak-Wien, Über die Beziehungen von Infektionskrankheiten zu normalen und krankhaften Yeränderungen des weíblichen Genitales.

Die Frage des Übergangs von Krankheitskeimen aus dem mutter-lichen in den kindlichen Organismus ist von hohem theoretischem und praktischem Interesse. Von nicht geringerer Bedeutung ist dieTJbertragung der Immunität durch die Plazenta und die Milch. Endlich ist von klini-scher Wichtigkeit der Einfluß, den Menstruation, Schwangerschaft und Wochenbett auf die Infektionskrankheiten und umgekehrt ausüben. Novak hat unsere heutigen Kenntnisse über diese 
Probleme für jede einzelne Infektionskrankheit an Hand der Literatur übersichtlich gesammelt und damit eine höchst willkommene Arbeit geleistet.

M. Weinberger-Wien, Beziehungen zwíschen Erkrankungen der Atmungs-organe und der weíblichen Generationsorgane.

Dieser Beitrag eines Internisten darf als wertvolle Ergänzung zu den zahlreichen Bearbeitungen dieses Themas von gynäkologischer Seite be-zeichnet werden. Tuberkulose, Grippe, Asthma, kruppöse Pneumonie, Lungenembolie werden in den Kreis der Erörterung gezogen. Einen breiten Raum nimmt die Frage der Schwangerschaftsunterbrechung wegen Tuberkulose ein.

Auch Weinberger steht, wie wohl die Mehrzahl der Cynäkologen, auf dem Standpunkt, daß bei aktiver florider Erkrankung der Lungen die Schwangerschaft in einer möglichst frühen Zeit zu unterbrechen ist. Die Hauptschwierigkeiten erblickt Weinberger in der sicheren Diagnose dieses Stadiums. Ebenso gründlich werden die Indikationen zur Sterilisation und die Frage der Stillerlaubnis bei tuberkulösen Müttern erörtert.

H. Schur-Wien, Stoffwechsel und Gijnäkologíe.

Der Internist Schur beginnt seine Ausführungen mit dem für tins besonders schmeichelhaften Satz, daß es wenig Sondergebiete der Medizin gäbe, in denen das Interesse für

Stoffwechselfragen so rege ist wie in dem der Gynäkologie. Um so mehr darf er unseres Dankes sicher sein für die überaus gründliche, vielfach auf eigenen wertvollen Untersuchungen beruhende Bearbeitung dieses großen und wichtiger) Kapitels. Der Stoffwechsel in der Schwangerschaft, bei der Laktation, Ovulation und Menstruation, nach Kastration und im Klimakterium sowie bei den versc $\cdot h i e d e n e n$

76

Buchbesprechungen.

Genitalerkrankungen ist mit ebenso gründlicher Sachkenntnis besprochen wie die Erkrankungen der Keimdrüsen und ihrer Funktionen als Folge von Änderungen des Stoffwechsels und der Einfluß von Stoffwechsel-störungen auf den Ablauf einer Schwangerschaft. Es ist unmöglich, in einem kurzen Referat die Ergebnisse seiner Arbeit auch nur annähernd erschöpfend zu würdigen, erstrecken sie sich doch auf die gesamten Ge-biete des Eiweiß-, Fett-, Kohlehydrat- und Mineralstoffwechsels und deren Abhängigkeit von den verschiedensten liormonalen Einflüssen, ein Gebiet, das, wie die Literaturzusammenstellung am Ende des Abschnittes zeigt, uiigeheuer verzv/eigt ist und der Erforschung außerordentliche Schwierig-keiten bereitet. Nur auf eine Schlußfolgerung Schurs sei aufmerksam ge-macht, die bei der Autorität des Verfassers auf diesem Gebiet besondere Beachtung verdient: Schur hebt mit Nachdruck hervor, daß die Hoffnungen, clurch Belebung der Geschlechtsdrüsen oder durch Implantation artgleicher oder ariverwandter Keimdrüsen eine Verjüngung mit Ver-längerung des Lebens zu erzielen, jeder realen Grundlage entbehren. Die Geschlechtsorgane dienen lediglich der Fortpflanzung, sind aber keine lebenswichtigen Organe. Scheiden sie im hohen Alter avis, so kann ihr künstlicher Ersatz wohl eine vorübergehende Sexualisierung, aber keine Lebensverlängerung der übrigen Körperzellen bewirken.

E. Schwarz-Wien, Die Beziehungen der Nebenniere zum weiblichen Ge-schlechísapparat. Die Beziehungen zwischen Nebenniere und Gesehlechtsorganen führen tief in das verwickelte Problem der Geschlechtlichkeit und der Inter -sexualität. Schwarz selbst sagt darüber: ,,Der Weg, den die Frage nach den Zusammenhéingen zwischen Nebenniere und weiblichem Geschlechts-apparat uns gehen läßt, führt von sicher physiologischen Tatsachen durch ein Wirrsal teils noch unzusammenhängender, teils sogar widerstreitender Feststellungen und Meinungen und endet in einem fast unbekannten Gebiet der menschlichen Konstitution, in 
welches nur aus fernen Lebens-bezirken Wegspuren hineinführen." ITm so mehr lockt es, sich mit diesen geheimnisumgebenen Fragen zu beschäftigen, besonders an der Hand eines so kundigen Führers wie Schwarz, dessen fesselnde Darstellungsweise licht-volle Orcinung in dieses Labyrinth zu bringen versteht.

Dersclbo, Beziehungen von MHz und Pankreas zum weiblichen Genitale.

Wesentlich einfacher als die genitalen Beziehungen der Nebenniere sind die der Milz und des Pankreas. Sie beschränken sich im Großen und Ganzen auf die seltenen Milzzerreißungen in der Schwangerschaft und unter der Geburt, den Einfluß der Milz auf die Gerinnung des Blutes (daher die Milz-Reizbestrahlungen bei funktionellen Genitalblutungen) und die Wandermilz, die ausschließlich eine Eigentümlichkeit des weiblichen Ge-schlechts ist.

Am Pankreas endlich kommen Zysten vor, die zu Verwechslung mit Ovarialkystomen Anlaß geben können.

W. Latzko-Wien und J. Sehiîfmann-Wien, Erkrankungen des weiblichen Harnapparats und ihre Beziehungen zu den weiblichen Genííalorganen. Mit Ausschluß der Gonorrhöe, der Fisteln und der nicht chirurgischen

Buchbesprechungen.

77

Nierenkrankheiten, die an anderen Stellen des Handbuchs besprochen sind, findet der Leser hier eine erschöpfende Darstellung der gynäko-logischen Urologie. Auf die Beschreibung der Untersuchungsmethoden, bei denen wir nur eine Berücksichtigung des Lî̀̈ysschen Zystoskops ver-missen, folgen die Erkrankungen der Harnröhre, der Blase, des Hamleiters, des Nierenbeckens und der Nieren. Zweckmäßig ist jedem einzelnen Ab-schnitt sofort ein ausführliches Literaturverzeichnis angefügfc. Sehr schön sind die farbigen und nichtfarbigen Abbildungen und Tafeln, die ja für das Verständnis besonders von Blasenerkrankungen geradezu unentbehr-lich sind, sehr schön auch die Wiedergabe von zahlreichen Röntgeno-grammen. Bei den großen persönlichen Erfahrungen Latzkos und Schiff-manns auf dem Gebiet der gynäkologischen Urologie nimmt man die da und dort etwas subjektiv gefärbte Darstellung gern in Kauf. Neben Stoeckels Bearbeitung dieses Gebiets in Veils Handbuch der Gynäkologie und in Döderleins Handbuch der Geburtshilfe, stellt diese Monographie der Wiener Autoren eine ebenbürtige Leistung dar. Es dürfte wohl kaum eine Situation goben, für die der gynäkologische Urologe hier nicht Auf-schluß und zuverlässigen Rat finden würde. Besondere Anerkennung verdient der anschauliehe flüssige Stil und die trotz des großen Umfangs niemals ermüdende Darstellung.

J. Novak-Wien, Beziehungen zwischen Nervensystem und Genitale.

Novaks Abhandlung beschränkt sich auf den wechselseitigen Einfluß zwischen organischen Nervenleiden und dem weiblichen Genitale, umfaßt aber auch in dieser Beschränkxmg noch ein beträchtliches Gebiet von größter praktischer Bedeutung. Vorausgeschickt ist das glänzend gesehriebene Kapitel über die Anatomie und Physiologie des weiblichen Genital-Nervenapparates, das mit seinen zahlreichen instruktiven Abbildungen einen vortrefflichen Einblick in Verlauf, Zusammenhang und Funktion des zerebrospinalen und vegetativen Nervensystems vermittelt. Es folgen Kapitel über den Einfluß der Pubertät, über Beziehungen zwischen Menstruation, Geschlechtsleben, Schwangerschaft, Geburt und Wochenbett zu den organischen Nervenleiden. Ausdrücklich hingewiesen sei auf die nun folgende zusammenhängende Darstellung der Schädigungen des kindlichen Nervensystems durch Geburtstraumen, speziell Gehirn-traumen. Von besonderem Interesse für den Geburtshelfer ist die Frage der Spätschädigungen, die nach einem Geburtstrauma des Gehirns auf-treten: Littlesehe Krankheit, Pseudobulbärparalyse, 
progressive Starre, zerebrale Kinderlähmung, Epilepsie, Chorea, Schwachsinn und Idiotie. Die Lektüre dieser Abschnitte ist jedem Geburtshelfer dringend zu empfehlen, da sie ihm, der sonst nur die Neugeborenen sieht, die Kennt-nisse und Beobachtungen erfahrener Kinderärzte vermittelt, die wir nicht unberücksichtigt lassen dürfen, und die für die Indikationen unseres geburtshilflichen Handelns von größter Bedeutung sind. Auch das nächste Kapitel über Eheverbot, Konzeptionsverhütung und Schwangerschafts-unterbrechting bei Nervenleiden verdient voile Beachtung. Die Frage der eugenetischen Indikation der Schwangerschaftsunterbrechung, an die fast alle Autoren nur mit großer Vorsicht herangehen, wird hier in wissen-schaftlich mianfechtbarer Weise abgelïandelt.

78

Buchbesprechungeu. - Personalien.

E. Neurath-Wien, Phijsiologie und Pathologie der Pubertät des weiblichen

Geschlechís.

Ein Handbucb. der Biologie des Weibes konnte an diesem nächst dem Klimakterium einschneidendsten Abschnitt des weiblichen Lebens nicht vorübergehen. Die Psychiater haben uns zuerst gelehrt, welch gewaltigen Einfliiß der Ablauf des Pubertätsalters für die psychische Entwicklung des Menschen besitzt. Eine systematische Bearbeittmg des Alters der Geschlechtsreife stand aber in der Literatur bisher noch aus. Die Neurathsche Arbeit, die sich bezüglich der somatischen Erscheinungen vielfach an die Straízscheií Untersuchungen, für die psychische Entwicklung an das Buch von Spranger, „Die Psyche des Jugendalters”, anlehnt, ist ein begrüßens-werter Versuch, diese Lücke auszufüllen, da Neurath die beiden Seiten, die körperliche und psychische, unter einheitlichem Gesichtswinkel ge-sehen, zusammenfaßt. Die Theorie der Pubertät, ihre Abhängigkeit von der ererbten Konstitution und von den Funktionen der endokrinen Drüsen und ihre allgemeine und spezielle Pathologie, vor allem die Pubertas praecox und tarda, werden eingehend gewürdigt. Im Schlußkapitel kommt die Hygiene des Pubertätsalters kurz, vielleicht zu kurz, zu Wort. Die Erwartungen Neuraths: Mutter und Vater werden am ehesten den liebe-und taktvollen Weg zum Verständnis der Kinder finden, vermögen wir nicht zu teilen, da Laien gerade den seelischen Eigentümlichkeiten der Pubertätszeit sehr häufig ohne jedes Verständnis gegenüberstehen.

K. Baisch. "V5/8iies, Henri: Phijsiologie gynécologíque et médecine des †emmes. Paris 1929. Masson \& Cie. 65 Fr.

Als Gegenstück zu seiner Physiologie der Fortpflanzung hat der Ver-fasser uns hier eine Darstellung der Funktionen des weiblichen Körpers außerhalb der mit der Fruchtbarkeit zusammenhängenden Fragen be-schert. Die großen biologischen Fragen werden an Hand der einzelnen Akte im Leben des Weibes entwickelt und dabei immer der große ganze Zusammenhang gewahrt. Die Beziehungen zwischen direkten und indirekten Geschlechtsmerkmalen vor und nach der Pubertät, die Ausbildung zu einem sexuellen Typ, die Wellenbewegungen im Leben der Frau und ihre Zu-sammenhänge mit dem vegetativen System humoralen und zellulären Charakters werden elegant abgehandelt. Das Geschlechtsleben während der Zeit der Ovulation und die klimakterischen Erscheinungen infolge spontaner Amenorrhöe oder artefizieller Sterilisierung bieten einen interes-santen Einblick in das biologische Geschehen. Die statischen Verhältnisse des Genitaltraktus bringen neuartige Befunde über den Schaden des Auto-fahrens. Die Bedeutung der ovariellen Inkretion und die Gesamtwirkung im System der endokrinen Drüsen schließen das Werk ab. Das Werk gibt einen guten Überblick über die Beziehungen zwischen Geschlechtsleben und allgemeiner Körperfunktion des weiblichen Menschen. 
G. H. Schneider -Brandenburg.

Personalienu

Privatdozent für Geburtshilfe und Gynäkologie Dr. Karl Herold in Jena ist zum nichtbeamteten a. o. Professor ernannt. 